\title{
Articles
}

\section{Changes in physical activity are associated with changes in metabolic cardiovascular risk factors}

\author{
L. Byberg $^{1}$, B.Zethelius ${ }^{1}$, P.M.McKeigue ${ }^{2}$, H. O. Lithell ${ }^{1}$ \\ ${ }^{1}$ Section of Geriatrics, Department of Public Health and Caring Sciences, Uppsala University, Uppsala, Sweden \\ ${ }^{2}$ Department of Epidemiology and Population Health, London School of Hygiene and Tropical Medicine, London, UK
}

\begin{abstract}
Aims/hypothesis. To investigate the effect of changes in physical activity on changes in metabolic cardiovascular risk factors and to investigate what factors affect the association between physical activity and cardiovascular mortality.

Methods. Of the 1860 men who were 50 years of age and who were without pre-existing cardiovascular disease participating in a population-based study, 898 were re-examined 20 years later. Altogether 231 died from cardiovascular diseases during the followup (mean $=22.6$ years). The examinations which the men underwent at 50 and 70 years of age included assessment of physical activity (self-reported at four alternative levels), anthropometry, measurements of fasting concentrations of glucose, specific insulin, proinsulin, split proinsulin and lipids.

Results. During the 20 years, $31 \%$ increased their amount of physical activity while $51 \%$ continued the same amount of exercise. Increased physical activity was associated with significant changes in several important metabolic variables, including fasting glu-
\end{abstract}

cose, proinsulin and HDL cholesterol, independent of body weight changes. The risk of cardiovascular disease for men performing moderate, regular and athletic physical activity was $25 \%(p=0.127), 34 \%$ $(p=0.022)$ and $71 \%(p=0.009)$ lower, respectively, compared with sedentary men. The association was attenuated by adjustment for baseline measurements of insulin, proinsulin and split proinsulin. Additional adjustment for other cardiovascular risk factors did not further attenuate the association.

Conclusion/interpretation. Increased leisure time physical activity between the ages of 50 and 70 years, in the absence of active intervention, is associated with improved glucose, insulin and lipid metabolism in men. The concentrations of insulin, proinsulin and split proinsulin could mediate much of the association between a sedentary lifestyle and increased risk of cardiovascular mortality. [Diabetologia (2001) 44: 2134-2139]

Keywords Physical activity, cardiovascular diseases, mortality, insulin resistance, proinsulin, longitudinal studies, risk factors, records.
Several studies have shown a beneficial effect of physical activity and exercise on health. A higher de-

Received: 6 July 2001 and in revised form: 23 August 2001

Corresponding author: L. Byberg, Section of Geriatrics, Department of Public Health and Caring Sciences, Uppsala University, P. O. Box 609, SE-75125, Uppsala, Sweden, e-mail: liisa. byberg@pubcare.uu.se

Abbreviations: CI, Confidence interval; HR, hazard ratio from Cox proportional hazard regression; s-CE, serum cholesterol ester. gree of or an increase in physical activity or physical fitness is associated with a lower risk of mortality or incidence of cardiovascular diseases [1-8], independent of several cardiovascular risk factors including smoking, obesity, blood pressure, serum lipids, socioeconomic status and diabetes [3-5].

Physical activity also has a beneficial effect on many cardiovascular risk factors [2]. A higher degree of physical activity has been associated with a lower incidence of Type-II (non-insulin-dependent) diabetes mellitus [9] and a lower degree of insulin resistance $[10,11]$. Fasting concentrations of (immunore- 
active) insulin, often used as a proxy measurement of insulin resistance, have been independently associated with cardiovascular mortality [12]. One previous study investigating the role of insulin - along with other metabolic risk factors such as serum lipids and obesity - in the effect of physical activity on mortality showed minor effects of metabolic factors on the association [13].

The aims of this population-based observational study were to assess the association between change in amounts of physical activity, in the absence of active intervention, and changes in metabolic factors, and also to examine the cross-sectional relationships between physical activity level and metabolic characteristics. Furthermore, we aimed to investigate which metabolic variables explain the possible relationship between physical activity and cardiovascular mortality.

\section{Subjects and methods}

The subjects in this study participated in the Uppsala Longitudinal Study of Adult Men (ULSAM) described previously [14-17]. All men in Uppsala born between 1920 and 1924 $(n=2841)$ were invited to a health investigations in 1970 , in which 2322 (82\%) participated. Participants still alive and living in Uppsala were traced by their ten-digit social security number and invited to another examination in 1991 $(n=1681)$, in which 1221 men $(73 \%)$ participated. During the intervening 20 years, 422 men had died and 219 had moved from Uppsala. Information on medical treatment and previous diseases was collected using a medical questionnaire. Information on incident disease was collected from the official Swedish in-patient, cancer, and cause-of-death registries. The study was approved by the local ethics committee and all participants gave their informed consent. All investigations were done after an overnight fasting.

Study population. Of the 232250 -year-old men, a total of 362 with cardiovascular, pulmonary or cancer diseases were excluded, based on the following exclusion criteria: previous myocardial infarction or angina; current treatment with nitroglycerine or digitalis; presence of Q or QS patterns (Minnesota codes 1.1-1.3) or left bundle branch block (Minnesota code 7.1) in the electrocardiogram examination at age 50 [14]; incident cardiovascular disease up to one year after baseline (ICD-9 codes: 390-399 and 410-449); previous tuberculosis, asthma or other chronic pulmonary problems up to one year after baseline (ICD-9 codes 490-519); and previous or incident malignant cancer up to two years after baseline (ICD-9 codes 140-208 and 230-239).

For another 100 of the 50-year-old men information on leisure time physical activity was missing, leaving 1860 men aged 50 years in the present study population. Of these men, 998 were re-examined at 70 years of age (223 of the 1221 men examined at 70 years of age were already excluded based on disease at age 50). For 100 of the remaining men, information on physical activity at 70 years of age was missing, leaving 898 men who were examined and who provided information on physical activity at both ages. The only difference in metabolic characteristics between men with missing information on physical activity and men included in the study was a higher
BMI at age 70 for men with missing information on physical activity at that age than the men included $\left(26.9 \mathrm{vs} 26.1 \mathrm{~kg} / \mathrm{m}^{2}\right.$, respectively; $p=0.006$ ).

Assessment of physical activity. Leisure time physical activity was assessed using four questions in the medical questionnaire. These were: 1) Do you spend most of your time reading, watching TV, going to the cinema or engaging in other, mostly sedentary, activities?, 2) Do you often go walking or cycling for pleasure?, 3) Do you engage in any active sport or heavy gardening for at least 3 hours every week? and 4) Do you regularly engage in hard physical training or competitive sport? Based on these questions, four physical activity categories were constructed: Sedentary (1), Moderate (2), Regular (3), and Athletic (4). The questionnaire was constructed such that each question could be answered 'yes' or 'no'. For example, to qualify for the Sedentary category, only the first question could be answered by 'yes' and to qualify for the Regular category, at least question number 3 ) had to be answered by 'yes', but the answer to the fourth question had to be 'no'. The physical activity categories used in this study have been used and validated by others $[5,18]$.

The medical questionnaire used for the men at 50 years of age also included questions on physical activity at work, which was categorised as 1) sedentary, 2) mostly standing and walking, 3) heavy lifts ( > $10 \mathrm{~kg}$ ), and 4) physically demanding work.

Clinical and metabolic investigations. Information on smoking habits was obtained through physician's interview; $51 \%$ (951 men) were regular smokers at 50 years of age. Information on education and marital status was collected from the Swedish census from 1970.

Investigations at 50 and 70 years of age included height $(\mathrm{m})$, weight $(\mathrm{kg})$, office supine blood pressure $(\mathrm{mmHg})$ and pulse rate (beats/min), fasting glucose (measured in blood at age 50 and in plasma at age 70) and serum lipids and lipoproteins, as described previously [14-17]. The BMI was calculated as the weight divided by the squared height $\left(\mathrm{kg} / \mathrm{m}^{2}\right)$. The concentrations of specific insulin, proinsulin and 32-33 split proinsulin were analysed in serum samples stored frozen at $-70^{\circ} \mathrm{C}$ until analysis was performed in 1995-98 using two-site immunometric assays [19]. The serum cholesterol ester fatty acid proportions were analysed using gas-liquid chromatography $[20,21]$. The ratio of oleic to linoleic acid $(18: 1 / 18: 2 \omega 6)$ was used as a marker of saturated and polyunsaturated fat intake [22].

Mortality follow-up. As of the end of 1996, 523 of the 1860 men in this study had died. According to the cause of death stated in the official Swedish cause-of-death registry, 194 deaths were due to malignant cancers (ICD-9 codes 140-208 and 230-239) and 231 to cardiovascular diseases (ICD-9 codes 390-459), of which 111 were acute myocardial infarctions (ICD-9 code 410 ) and 44 other ischaemic heart diseases (ICD-9 codes 411-414).

Statistical analyses. Statistical analyses were performed using Stata statistical software (Stata Corporation, College Station, Tex., USA). Variables are presented as arithmetic means and standard deviations (SD) and their associations with physical activity level were tested using Spearman's partial rank correlation and adjusted for age and BMI. Group comparisons of metabolic characteristics were performed using ANOVA. The effect of changes in physical activity on changes in metabolic variables was analysed using linear regression and is presented as relative change (\%) when physical activity is increased one step between 50 and 70 years of age. The dependent variable 
Table 1. Prevalence in the physical activity categories

\begin{tabular}{|c|c|c|c|c|}
\hline \multirow{2}{*}{$\begin{array}{l}\text { Physical activity } \\
\text { category }\end{array}$} & \multicolumn{2}{|l|}{50 years } & \multicolumn{2}{|l|}{70 years } \\
\hline & Prevalence (\%) & $n$ & Prevalence (\%) & $n$ \\
\hline Sedentary & 14.6 & 271 & 3.9 & 35 \\
\hline Moderate & 36.1 & 672 & 33.4 & 300 \\
\hline Regular & 44.4 & 825 & 57.0 & 512 \\
\hline Athletic & 4.9 & 92 & 5.7 & 51 \\
\hline
\end{tabular}

in the regression model was the natural logarithm (due to a skewed distribution) of the metabolic variable at 70 years of age and independent variables were the natural logarithm of the metabolic variable at 50 years of age, physical activity at 50 years of age and physical activity at 70 years of age. The relative change (\%) of the metabolic variable was then calculated as $\left(e^{b}-1\right)$, where $b$ is the regression coefficient for physical activity at 70 years of age from the linear regression model described above, and e is the base of the natural logarithms. The relations between physical activity and different mortality outcomes were tested using Cox proportional hazard regression analysis. The results are presented for each physical activity category as hazard ratios (HR) and their $95 \%$-confidence intervals (CI), using the lowest physical activity category as reference. The mean follow-up on mortality in this study was 22.6 years $(\min =0.04$ and $\max =26.7$ years $)$. A $p$ value of less than 0.05 was regarded as statistically significant.

\section{Results}

The distribution of men at the age of 50 and 70, respectively, in the physical activity categories, is presented in Table 1 . The amount of physical activity was inversely associated with BMI, and the relations to the other metabolic characteristics investigated were therefore adjusted for BMI. A higher amount of physical activity was associated with a more favourable metabolic profile both at 50 years of age and at 70 years of age, independent of BMI (Table 2).

The amount of physical activity during leisure time was not associated with the amount of physical activity done at work.

Fifty-one per cent $(n=454)$ of the 898 men who participated in both investigations reported no change in the amount of physical activity during the 20 years, whereas $31 \%$ had increased $(n=282)$ and $18 \%$ had decreased $(n=162)$ their amount of physical activity, as shown in detail in Table 3 . There were no differences between these groups regarding the metabolic variables measured when the men were 50 years of age. The change in leisure time, but not work time physical activity between investigations was associated with concomitant changes in metabolic variables, shown in Table 4 . With each increasing level of physical activity, body weight significantly decreased by one per cent, hence associations with changes in other metabolic variables were adjusted for weight changes. The largest relative change associated with a change in physical activity was the concentrations of 32-33 split proinsulin, which decreased by $10 \%$ with each increasing level of physical activity. The associations did not change after adjustment for hospital treatment of cardiovascular diseases, treatment with beta-blocking drugs or physical immobility.

Men who reported moderate physical activity had a $25 \%$ lower risk of cardiovascular mortality compared with sedentary individuals, although it was not statistically significant (HR: $0.75,95 \%-C I$ : $0.52-1.08)$. Regular physical activity was associated with a lower risk of cardiovascular mortality of $34 \%$

Table 2. Means of metabolic variables and their association with physical activity, adjusted for age and BMI, in men who were 50 and 70 years of age

\begin{tabular}{|c|c|c|c|c|c|c|}
\hline & \multicolumn{3}{|l|}{50 years } & \multicolumn{3}{|l|}{70 years } \\
\hline & Mean (SEM) & $n$ & $r$ & Mean (SEM) & $n$ & $r$ \\
\hline Weight $^{\mathrm{b}}(\mathrm{kg})$ & $77.6(10.9)$ & 1860 & -0.02 & $79.9(11.1)$ & 898 & -0.06 \\
\hline Systolic blood pressure (mmHg) & $133(18)$ & 1859 & -0.01 & $147(18)$ & 896 & -0.01 \\
\hline Diastolic blood pressure (mmHg) & $83(11)$ & 1859 & -0.001 & $84(9)$ & 896 & 0.02 \\
\hline Pulse rate (beats/min) & $69(11)$ & 1860 & $-0.09^{\mathrm{a}}$ & $65(9)$ & 891 & $-0.11^{\mathrm{a}}$ \\
\hline Serum triglycerides $(\mathrm{mmol} / \mathrm{l})$ & $1.91(1.19)$ & 1860 & $-0.09^{\mathrm{a}}$ & $1.43(0.73)$ & 898 & $-0.08^{\mathrm{a}}$ \\
\hline LDL cholesterol $(\mathrm{mmol} / \mathrm{l})$ & $5.28(1.26)$ & 1526 & $-0.05^{\mathrm{a}}$ & $3.90(0.89)$ & 894 & 0.05 \\
\hline LDL/HDL cholesterol ratio & $4.21(1.76)$ & 1526 & $-0.08^{\mathrm{a}}$ & $3.22(1.06)$ & 894 & -0.03 \\
\hline Fasting glucose $\mathrm{c}^{\mathrm{c}}(\mathrm{mmol} / \mathrm{l})$ & $5.0(0.8)$ & 1854 & -0.02 & $5.8(1.5)$ & 898 & -0.05 \\
\hline Specific insulin $(\mathrm{pmol} / \mathrm{l})$ & $50.1(38.6)$ & 1045 & $-0.07^{\mathrm{a}}$ & $51.6(37.9)$ & 869 & $-0.09^{\mathrm{a}}$ \\
\hline Proinsulin $(\mathrm{pmol} / \mathrm{l})$ & $3.1(3.3)$ & 1056 & $-0.07^{\mathrm{a}}$ & $8.5(8.8)$ & 861 & $-0.12^{\mathrm{a}}$ \\
\hline 32-33 Split proinsulin $(\mathrm{pmol} / \mathrm{l})$ & $7.4(7.2)$ & 1032 & $-0.13^{\mathrm{a}}$ & $10.9(11.3)$ & 861 & $-0.10^{\mathrm{a}}$ \\
\hline
\end{tabular}

${ }^{\mathrm{a}} p<0.05$

${ }^{\mathrm{b}} r$ adjusted for age only

${ }^{c}$ Fasting glucose was measured in blood at age 50 and in plasma

$r$ indicates Spearman's partial correlation coefficient, adjusted for age and BMI; s-CE serum cholesterol ester 
Table 3. Change in physical activity from 50 to 70 years, by physical activity category at age 50

\begin{tabular}{|c|c|c|c|c|c|}
\hline \multirow[t]{2}{*}{ Physical activity level at age 50} & \multicolumn{5}{|c|}{ Change in physical activity (50 to 70 years), \% ( $n$ ) } \\
\hline & -2 & -1 & 0 & +1 & +2 \\
\hline Sedentary & - & - & $7.6(8)$ & $45.7(48)$ & $46.7(49)$ \\
\hline Regular & $3.5(15)$ & $23.4(101)$ & $66.0(285)$ & $7.2(31)$ & - \\
\hline Athletic & $13.7(7)$ & $56.9(29)$ & $29.4(15)$ & - & - \\
\hline Total & $2.5(22)$ & $15.6(140)$ & $50.6(454)$ & $25.5(229)$ & $5.9(53)$ \\
\hline
\end{tabular}

Table 4. Relative change in metabolic variables when increasing physical activity one step between age 50 and age 70 years

\begin{tabular}{|c|c|c|c|c|c|}
\hline & \multirow[b]{2}{*}{$n$} & \multicolumn{2}{|l|}{ Crude } & \multicolumn{2}{|c|}{ Adjusted for weight change } \\
\hline & & Change (\%) & $p$ & Change (\%) & $p$ \\
\hline Weight & 898 & -1.1 & 0.018 & - & - \\
\hline Pulse rate & 891 & -1.6 & 0.016 & -1.5 & 0.019 \\
\hline Serum triglycerides & 898 & -5.4 & 0.010 & -3.5 & 0.078 \\
\hline Serum cholesterol & 898 & +1.2 & 0.143 & +1.5 & 0.068 \\
\hline HDL cholesterol & 726 & +4.6 & 0.001 & +4.2 & 0.002 \\
\hline Fasting glucose & 895 & -2.3 & 0.017 & -2.0 & 0.040 \\
\hline Specific insulin & 405 & -7.2 & 0.072 & -3.5 & 0.337 \\
\hline Proinsulin & 400 & -9.8 & 0.016 & -7.4 & 0.057 \\
\hline $32-33$ split proinsulin & 390 & -14.6 & 0.002 & -10.0 & 0.023 \\
\hline s-CE-oleic/linoleic acid ratio $(18: 1 / 18: 2 \omega 6)$ & 418 & -1.8 & 0.186 & -1.4 & 0.319 \\
\hline
\end{tabular}

s-CE, serum cholesterol ester

Table 5. Hazard ratios (95\%-confidence intervals) for cardiovascular mortality according to leisure time physical activity category at baseline, adjusted for baseline characteristics

\begin{tabular}{lllll}
\hline Physical activity category & A & B & C & D \\
\cline { 2 - 5 } & HR $(95 \%-C I)$ & HR $(95 \%-C I)$ & HR $(95 \%-C I)$ & 1.00 \\
Sedentary & 1.00 & 1.00 & $0.72(0.47-1.12)$ & 1.00 \\
Moderate & $0.62(0.40-0.96)$ & $0.67(0.43-1.04)$ & $0.76(0.50-1.16)$ & $0.67(0.43-1.04)$ \\
Regular & $0.60(0.40-0.91)$ & $0.68(0.44-1.03)$ & $0.25(0.06-1.04)$ & $0.25(0.47-1.10)$ \\
Athletic & $0.16(0.04-0.66)$ & $0.19(0.05-0.81)$ & $-1.05)$ \\
\hline
\end{tabular}

Total $n=880, n$ cases $=153$

A, age-adjusted; B, adjusted for age, smoking, marital status, education, physical activity at work, serum cholesterol ester oleic/linoleic acid ratio (18:1/18:2 $\omega 6)$; $\mathrm{C}$, adjusted for B and

(HR: 0.66, 95\%-CI: 0.46-0.94) and the men in the athletic group had a reduced risk of $71 \%$ (HR: 0.29, 95\%-CI: $0.12-0.74)$. Similarly, the risk of all-cause mortality was $16 \%(p=0.173), 26 \%(p=0.016)$ and $54 \%(p=0.004)$ lower in the moderate, regular and athletic physical activity groups, respectively, compared to the sedentary group. Additional exclusion of men taking anti-hypertensive medication or men with joint or lung problems did not change the association between physical activity and mortality.

Adjustment for socio-economic and lifestyle cardiovascular risk factors attenuated the relations between physical activity and cardiovascular mortality somewhat (Table 5). Additional adjustment for insu- for specific insulin, proinsulin, split proinsulin; $\mathrm{D}$, adjusted for $\mathrm{C}$ and for BMI, fasting blood glucose, serum triglycerides and total, low-density lipoprotein and high-density lipoprotein cholesterol

lin, proinsulin and split proinsulin turned the relation non-significant. Adding other cardiovascular risk factors, including factors associated with the insulin resistance syndrome and total and LDL cholesterol, did not further change the relations.

\section{Discussion}

This study shows that a higher level of physical activity is associated with both a more beneficial metabolic profile and a lower risk of mortality from cardiovascular diseases. The study also shows that the factors that mediate much of the association between 
physical activity and mortality, i.e. insulin and proinsulin, are those that over time showed the greatest improvement associated with an increase in physical activity.

It has been shown that exercise reduces the plasma concentrations of triglycerides and increases the concentrations of HDL cholesterol [11]. A change in proinsulin concentrations could reflect a change in insulin sensitivity [23, 24]. Insulin sensitivity and glucose disposal are improved by exercise [11].

Earlier longitudinal observational studies have shown that changes in physical activity have an effect on obesity and lipids [25]. No such previous study has included measurements of all three of glucose, insulin and proinsulin concentrations. Results from the present investigation imply that a moderately large increase in leisure time physical activity could improve the main features of the insulin resistance syndrome, independent of weight changes. However, as concomitant changes in physical activity and metabolic parameters are studied, no strict conclusions regarding the causality can be drawn.

Changes in physical activity were not associated with changes in the oleic-to-linoleic acid ratio of serum cholesterol esters (considered a marker of saturated and polyunsaturated fat intake in a typical western diet [22, 26]). The associations between changes in physical activity and changes in metabolic factors were also adjusted for changes in weight. This adjustment could take some dietary differences into account. On the other hand, increased physical activity is in itself associated with reduced weight and amount of intra-abdominal fat, followed by changes in plasma lipids and insulin sensitivity [11]. Thus, the true associations between physical activity and metabolic factors could be reduced by adjustment for weight.

Almost one-third of the men increased their physical activity between 50 and 70 years of age. Thus, ageing does not necessarily imply a reduced amount of physical activity. The participants did not receive any active recommendations about increasing their physical activity. During the 20 years, the men in the study reached the official age of retirement (65 years) and the increased spare time could largely have contributed to the increased physical activity. The same men answered the same set of questions at baseline and twenty years later, reducing the risk of assessment bias. However, the categories indexing leisure time physical activity should be regarded as reflecting common physical activity perceptions or patterns rather than precise measures of physical activity or exercise [27].

To reduce confounding due to co-morbidity in the mortality analyses, participants in the first examination with signs of cardiovascular, pulmonary or cancer diseases were excluded. Physical activity was inversely associated with mortality from cardiovascular diseases (and all causes). The association was weakened when adjusted for insulin, proinsulin and split proinsulin, factors related with insulin resistance [23, 24], indicating that these metabolic factors could mediate the higher risk of mortality among sedentary individuals.

In contrast to our results, one previous study investigating the role of insulin in the association between physical activity and cardiovascular disease could not show an effect of insulin or other metabolic factors [13]. Methodological differences between the two studies include measurements of non-fasting insulin compared with fasting insulin and proinsulin and physician's diagnosis of coronary heart disease compared with official registry data on cardiovascular mortality in the previous compared with the present study, respectively. The discrepant results could also be interpreted as indicating that proinsulin, and not insulin, is the more important factor mediating the association between physical activity and cardiovascular disease.

In a preliminary report made on analyses in this cohort, proinsulin predicts cardiovascular disease mortality and morbidity independent of other known cardiovascular risk factors [28]. It is not known whether proinsulin is atherogenic in itself [29], or is merely a better marker of insulin resistance [23, 24].

We conclude that an increased leisure time physical activity between 50 and 70 years of age, in the absence of active intervention, is associated with improved glucose, insulin, proinsulin and lipid metabolism in men. Insulin and proinsulin seem to be important factors that mediate much of the association between a sedentary lifestyle and increased risk of cardiovascular mortality.

Acknowledgements. We thank Professor C.N. Hales and his staff at the Department of Clinical Biochemistry, University of Cambridge, UK, for determining the concentrations of specific insulin, proinsulin and 32-33 split proinsulin. This work was supported by grants from Ernfors Fund for Diabetes Research, the Foundation for Geriatric Research, "Förenade Liv" Mutual Group Life Insurance Company, the Medical Faculty at Uppsala University, the Swedish Council for Planning and Co-ordination of Research (No. A19-5/62), the Swedish Diabetes Association, the Swedish Medical Research Council (No. 5446), the Swedish National Association Against Heart and Lung Disease and Trygg Hansa Research Fund.

\section{References}

1. Paffenbarger RS Jr, Hyde RT, Wing AL, Hsieh CC (1986) Physical activity, all-cause mortality, and longevity of college alumni. N Engl J Med 314: 605-613

2. Chandrashekhar Y, Anand IS (1991) Exercise as a coronary protective factor. Am Heart J 122: 1723-1739

3. Sandvik L, Erikssen J, Thaulow E, Erikssen G, Mundal R, Rodahl K (1993) Physical fitness as a predictor of mortality among healthy, middle-aged Norwegian men. N Engl J Med 328: 533-537 
4. Lakka TA, Venäläinen JM, Rauramaa R, Salonen R, Tuomilehto J, Salonen JT (1994) Relation of leisure-time physical activity and cardiorespiratory fitness to the risk of acute myocardial infarction in men. N Engl J Med 330: 1549-1554

5. Rosengren A, Wilhelmsen L (1997) Physical activity protects against coronary death and deaths from all causes in middle-aged men. Evidence from a 20-year follow-up of the Primary Prevention Study in Göteborg. Ann Epidemiol 7: 69-75

6. Paffenbarger RS Jr, Hyde RT, Wing AL, Lee IM, Jung DL, Kampert JB (1993) The association of changes in physicalactivity level and other lifestyle characteristics with mortality among men. N Engl J Med 328: 538-545

7. Blair SN, Kohl HWR, Barlow CE, Paffenbarger RS Jr, Gibbons LW, Macera CA (1995) Changes in physical fitness and all-cause mortality. A prospective study of healthy and unhealthy men. JAMA 273: 1093-1098

8. Wannamethee S, Shaper A, Walker M (1998) Changes in physical activity, mortality, and incidence of coronary heart disease in older men. Lancet 351: 1603-1608

9. Albright A, Franz M, Hornsby G et al. (2000) Exercise and type 2 diabetes. Med Sci Sports Exerc 32: 1345-1360

10. Mayer-Davis EJ, D'Agostino R Jr, Karter AJ et al. (1998) Intensity and amount of physical activity in relation to insulin sensitivity. The Insulin Resistance Atherosclerosis Study. JAMA 279: 669-674

11. Shahid SK, Schneider SH (2000) Effects of exercise on insulin resistance syndrome. Coron Artery Dis 11: 103-109

12. Ruige JB, Assendelft WJJ, Dekker JM, Kostense PJ, Heine RJ, Bouter LM (1998) Insulin and risk of cardiovascular disease. A meta-analysis. Circulation 97: 996-1001

13. Wannamethee SG, Shaper AG, Alberti KG (2000) Physical activity, metabolic factors, and the incidence of coronary heart disease and type 2 diabetes. Arch Intern Med 160: 2108-2116

14. Hedstrand H (1975) A study of middle-aged men with particular reference to risk factors for cardiovascular disease. Ups J Med Sci Suppl 19: 1-61

15. Skarfors ET, Selinus KI, Lithell HO (1991) Risk factors for developing non-insulin dependent diabetes: a 10 year follow up of men in Uppsala. BMJ 303: 755-760

16. Skarfors ET, Lithell HO, Selinus I (1991) Risk factors for the development of hypertension: a 10-year longitudinal study in middle-aged men. J Hypertens 9: 217-223

17. Byberg L, Siegbahn A, Berglund L, McKeigue P, Reneland R, Lithell H (1998) Plasminogen activator inhibitor-1 activity is independently related to both insulin sensitivity and serum triglycerides in 70-year-old men. Arterioscler Thromb Vasc Biol 18: 258-264
18. Løchen M-L, Rasmussen K (1992) The Troms $\varnothing$ study: physical fitness, self reported physical activity, and their relationship to other coronary risk factors. J Epidemiol Community Health 26: 103-107

19. Sobey WJ, Beer SF, Carrington CA et al. (1989) Sensitive and specific two-site immunoradiometric assays for human insulin, proinsulin, 65-66 split and 32-33 split proinsulins. Biochem J 260: 535-541

20. Öhrvall M, Berglund L, Salminen I, Lithell H, Aro A, Vessby B (1996) The serum cholesterol ester fatty acid composition but not the serum concentration of alpha tocopherol predicts the development of myocardial infarction in 50-year-old men: 19 years follow-up. Atherosclerosis 127: $65-71$

21. Vessby B, Tengblad S, Lithell H (1994) Insulin sensitivity is related to the fatty acid composition of serum lipids and skeletal muscle phospholipids in 70-year-old men. Diabetologia 37: 1044-1050

22. Glatz JF, Soffers AE, Katan MB (1989) Fatty acid composition of serum cholesteryl esters and erythrocyte membranes as indicators of linoleic acid intake in man. Am J Clin Nutr 49: 269-276

23. Phillips DIW, Clark PM, Hales CN, Osmond C (1994) Understanding oral glucose tolerance: comparison of glucose or insulin measurements during the oral glucose tolerance test with specific measurements of insulin resistance and insulin secretion. Diabet Med 11: 286-292

24. Mykkänen L, Zaccaro DJ, Hales CN, Festa A, Haffner SM (1999) The relation of proinsulin and insulin to insulin sensitivity and acute insulin response in subjects with newly diagnosed Type II diabetes: the Insulin Resistance Atherosclerosis Study. Diabetologia 42: 1060-1066

25. Thune I, Njølstad I, Løchen M-L, Førde OH (1998) Physical activity improves the metabolic risk profiles in men and women. The Troms $\varnothing$ Study. Arch Intern Med 158: 1633-1640

26. Sundstrom J, Lind L, Vessby B, Andren B, Aro A, Lithell HO (2001) Dyslipidemia and an Unfavorable Fatty Acid Profile Predict Left Ventricular Hypertrophy 20 Years Later. Circulation 103: 836-841

27. Haskell WL, Leon AS, Caspersen CJ et al. (1992) Cardiovascular benefits and assessment of physical activity and physical fitness in adults. Med Sci Sports Exerc 24: S201S220

28. Zethelius B, Lithell H, Hales CN, Berne C (1999) Proinsulin, a risk factor for cardiovascular disease. Diabetologia 42 [Suppl 1] I-IV: A5(Abstract)

29. Båvenholm P, Proudler A, Tornvall P et al. (1995) Insulin, intact and split proinsulin, and coronary artery disease in young men. Circulation 92: 1422-1429 